\title{
Psoriasis in Patients With Active Lung Cancer: Is Apremilast a Safe Option?
}

\author{
Zoe Apalla ${ }^{1}$, Eftychios Psarakis $^{1}$, Aimilios Lallas ${ }^{2}$, Alexia Koukouthaki ${ }^{1}$, \\ Athanassios Fassas ${ }^{3}$, Maria Smaragdi ${ }^{1}$
}

1 State Dermatology and Venereology Department, Hippokration Hospital, Thessaloniki, Greece
2 First Dermatology Department, Aristotle University of Thessaloniki, Greece
3 Oncology Department, St. Luke’s Private Hospital, Thessaloniki, Greece

Key words: psoriasis, adenocarcinoma, apremilast, phosphodiesterase inhibitor, treatment

Citation: Apalla Z, Psarakis E, Lallas A, Koukouthaki A, Fassas A, Smaragdi M. Psoriasis in patients with active lung cancer: is apremilast a safe option? Dermatol Pract Concept. 2019;9(4):300-301. DOI: https://doi.org/10.5826/dpc.0904a11

Accepted: May 22, 2019; Published: October 31, 2019

Copyright: @2019 Apalla et al. This is an open-access article distributed under the terms of the Creative Commons Attribution License, which permits unrestricted use, distribution, and reproduction in any medium, provided the original author and source are credited.

Funding: None.

Competing interests: The authors have no conflicts of interest to disclose.

Authorship: All authors have contributed significantly to this publication.

Corresponding author: Zoe Apalla, MD, PhD, 124 Delfon str, PC 54643, Thessaloniki, Greece. Email: zoimd@yahoo.gr

\section{Introduction}

Concomitant cancers in psoriasis patients often pose significant limitations in treatment decision. In contrast to the majority of the available systemic antipsoriatic drugs, apremilast is not contraindicated in case of previous or concomitant malignancy [1]. We report a patient with psoriasis and lung adenocarcinoma who completely responded to apremilast. The patient provided written informed consent for the data reported in the current manuscript.

\section{Case Presentation}

A 67-year-old woman visited the outpatient unit for an exacerbation of psoriasis (Psoriasis Area and Severity Index [PASI] score at baseline visit: 18). In 2013 she received a diagnosis of lung adenocarcinoma, stage IIIB. Since then she received various treatments, including pemetrexed, carboplatin, cisplatin, and radiotherapy. Progressive disease with liver metastases was detected in 2016. The patient received 6 courses of bevacizumab/docetaxel/carboplatin with documented partial response, and then nivolumab was started. The treatment was complicated by bacteremia. Antibiotics resulted in a drug reaction successfully managed with systemic steroids. Systemic drugs were discontinued, including nivolumab, despite an almost complete resolution of her metastatic disease. Tapering of steroids led to exacerbation of psoriasis.

Clinical examination revealed symmetrically distributed, pruritic, scaly plaques (Figure 1, A and B). The patient was not a good candidate for acitretin. After a thorough examination of her medical profile and in close collaboration with the oncologists, we decided to prescribe systemic apremilast (30 $\mathrm{mg} \times 2$, after dose titration from day 1 to day 5). The patient achieved PASI-75 in 16 weeks (PASI score at week 16: 4) (Figure 1, C and D). Nivolumab was concomitantly reinitiated, resulting in a documented response after 5 months. Based on the results of imaging examinations, the laboratory tests, and the overall assessment by an oncologist, there was no indication that the course of the cancer or the response to nivolumab was influenced by apremilast treatment. After 12 months of treatment with apremilast, the patient remains free of psoriasis recurrence. 


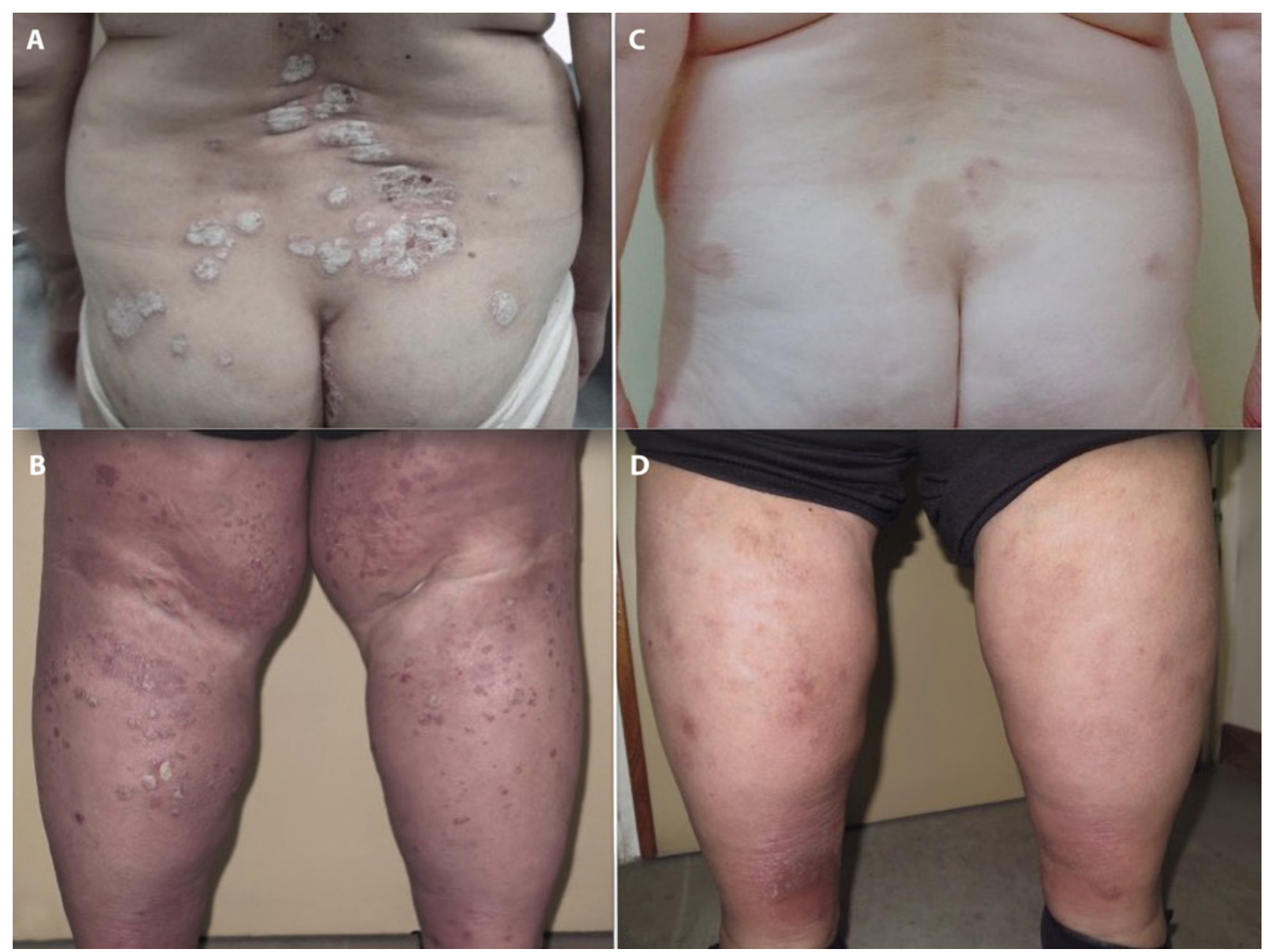

Figure 1. Erythematous, scaly plaques involving the lower back (A) and both legs (B). Complete clearance 16 weeks after apremilast initiation (C,D). [Copyright: @2019 Apalla et al.]

Evidence on the safety of small molecules in the context of psoriatic patients with known concomitant malignancies is poor. Apremilast is considered one of the safest options for the treatment of psoriasis. However, exclusion of patients with known malignancies from clinical trials does not allow safe conclusions for patients with solid organ malignancies.

We decided to use apremilast based on the lack of contraindication, as per summary of product characteristics. Furthermore, we were encouraged to follow this decision based on data about the pathophysiological role of phosphodiesterase 4 (PDE4) in lung and other cancer, at a cellular level. Analytically, in a previous study, investigators exposed different lung cancer cell lines to hypoxia and measured the activity and expression of PDE4. Interestingly, they found that PDE4 is expressed in lung cancer cells, cross-talks with hypoxia-inducible factor signaling, and promotes lung cancer progression. Based on this observation, they suggested that it could represent a potential therapeutic target [2]. Whether a similar effect would occur in vivo remains to be investigated further. However, the reported effect on cell lines indicates that PDE4 inhibition is quite likely—at the very least- to be harmless when given in patients with psoriasis and concomitant lung adenocarcinoma.

\section{Conclusions}

We report a favorable response of psoriatic lesions to apremilast in a patient suffering from metastatic lung adenocarcinoma, without any adverse event. Definite conclusions cannot be extracted before further studies confirm the safety of apremilast in a larger number of patients with concurrent malignancies.

\section{References}

1. Paul C, Cather J, Gooderham M, et al. Efficacy and safety of apremilast, an oral phosphodiesterase 4 inhibitor, in patients with moderate-to-severe plaque psoriasis over 52 weeks: a phase III, randomized controlled trial (ESTEEM 2). Br J Dermatol. 2015;173(6):1387-1399.

2. Pullamsetti SS, Banat GA, Schmall A, et al. Phosphodiesterase-4 promotes proliferation and angiogenesis of lung cancer by crosstalk with HIF. Oncogene. 2013;32(9):1121-1134. 